\title{
Latest Advances in Transcatheter Mitral Valve Replacement
}

\author{
Tomoya T Hinohara, ${ }^{1}$ Michael J Reardon ${ }^{2}$ and Sachin S Goel ${ }^{1}$ \\ 1. Department of Cardiology, Houston Methodist Hospital, Houston, TX, USA; 2. Department of Cardiovascular Surgery, DeBakey Heart \&
} Vascular Center, Houston Methodist Hospital, Houston, TX, USA

DOI: https://doi.org/10.17925/HI.2021.15.2.79

$\mathrm{M}$ itral regurgitation (MR) is the most prevalent valvular heart disease globally. Mitral valve surgery is the gold-standard treatment for MR. However, a significant portion of patients with mitral valve disease are at high or prohibitive surgical risk. Transcatheter mitral valve replacement (TMVR) has emerged as a potential treatment option for this vulnerable population. Numerous TMVR devices are currently being investigated, with early data demonstrating feasibility and efficacy of TMVR. In this article, we explore the unique challenges of designing a TMVR system and describe the TMVR systems under clinical evaluation.

\section{Keywords}

Mitral regurgitation, transcatheter mitral valve replacement, transcatheter valve therapies, mitral valve replacement, mitral valve surgery

Disclosures: Tomoya T Hinohara has no financial or non-financial relationships or activities to declare in relation to this article. Michael J Reardon is a consultant for Medtronic and serves on the executive committee of the APOLLO trial. Sachin $\mathrm{S}$ Goel serves on a speaker's bureau for Abbott Structural Heart and is a consultant for Medtronic.

\section{Review process: Double-blind peer review.}

Compliance with ethics: This study involves a review of the literature and did not involve any studies with human or animal subjects performed by any of the authors.

Data availability: Data sharing is not applicable to this article as no datasets were generated or analysed during the current study/during the writing of this article.

Authorship: The named authors meet the International Committee of Medical Journal Editors (ICMJE) criteria for authorship of this manuscript, take responsibility for the integrity of the work as a whole, and have given final approval for the version to be published.

Access: This article is freely accessible at touchCARDIO.com. (C) Touch Medical Media 202

Received: 11 October 2021

Accepted: 25 November 2021

Published online: 20 December 2021

Citation: Heart International. 2021;15(2):79-83

Corresponding author: Sachin S Goel,

6550 Fannin St, Suite 18.53, Houston 77030,

TX, USA. E: ssgoel@houstonmethodist.org

Support: No funding was received for the publication of this article.
Transcatheter-based therapies have become central to the treatment of valvular heart disease, particularly aortic stenosis. ${ }^{1-5}$ The first transcatheter aortic valve replacement (TAVR) was performed in 2002 and, in two short decades, TAVR has become a mainstay for the treatment of aortic stenosis for all surgical risk categories. ${ }^{1,6-8}$ With the global success and adoption of TAVR, significant efforts have been made to develop a transcatheter mitral valve replacement (TMVR) system for mitral regurgitation (MR).

MR is the most prevalent form of valvular heart disease worldwide. ${ }^{9,10}$ Approximately $10 \%$ of patients older than 75 years of age have at least moderate MR., ${ }^{910}$ Moreover, a longer life expectancy and a higher prevalence of ischaemic cardiomyopathy has led to a further increase in the prevalence of MR. ${ }^{11}$ Many of these patients are elderly with numerous comorbidities and are not ideal candidates for surgical mitral valve repair or replacement. This has created a large unmet need for an alternative, less invasive treatment option in patients who are at high or prohibitive surgical risk. ${ }^{12}$ Mitral transcatheter edge-to-edge repair (TEER) is currently utilized for patients at high surgical risk who remain symptomatic despite medical therapy. However, many patients are anatomically suboptimal for mitral TEER, including those with a mitral valve orifice area $<4.0 \mathrm{~cm}^{2}$, an elevated mean transmitral pressure gradient, insufficient leaflet length, calcification in the grasping area, presence of a significant cleft and leaflet perforation. . $13,14^{14}$

Treatment of MR depends on the aetiology of the MR. Primary or degenerative MR is a disease of the mitral valve apparatus and surgery is the gold-standard treatment." In patients at high or prohibitive surgical risk, TEER is also an approved therapy. ${ }^{1,13}$ In contrast, secondary or functional MR is a disease of the atria or ventricle, resulting in MR despite a structurally normal valve. The management of secondary MR is more complex, and guideline-directed medical therapy (GDMT) for heart failure and cardiac resynchronization therapy are the cornerstones of treatment. ${ }^{1}$ These therapies are aimed at treating the underlying pathology of cardiomyopathy and reversing adverse left ventricular remodelling. Surgical intervention for secondary MR does not improve clinical outcomes compared with medical therapy, and is limited to patients who have severe persistent symptoms despite optimal GDMT. ${ }^{1}$ Transcatheter mitral annuloplasty devices, including Cardioband (Edwards Lifesciences Corp., Irvine, CA, USA) and Carillon (Cardiac Dimensions, Inc., Kirkland, WA, USA) have been shown to effectively reduce secondary MR through annular reduction in feasibility studies, but the data are limited and these devices are not approved for commercial use in the USA. ${ }^{15-17}$ More recently, TEER has emerged as an effective therapy in patients with secondary MR based on the Cardiovascular outcomes assessment of the MitraClip percutaneous therapy for heart failure patients with functional mitral regurgitation (COAPT; ClinicalTrials.gov identifier: NCT01626079) trial. ${ }^{14}$ Treatment with TEER is a class 2a recommendation in society guidelines for patients who are symptomatic despite GDMT, while surgery is a class $2 \mathrm{~b}$ recommendation. ${ }^{1}$

The objectives of this review are to provide an overview of the challenges of developing TMVR devices and to describe the characteristics of different TMVR devices under clinical evaluation and their main clinical results. 
A
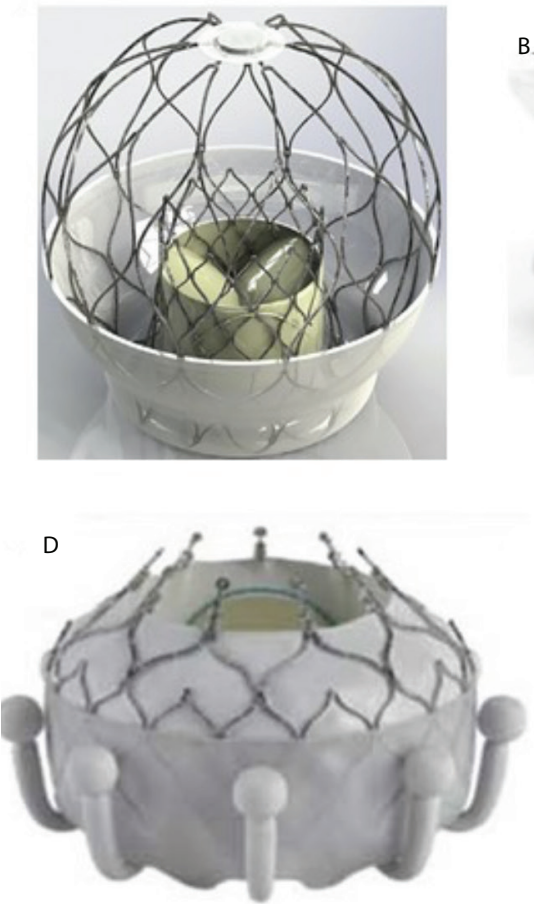

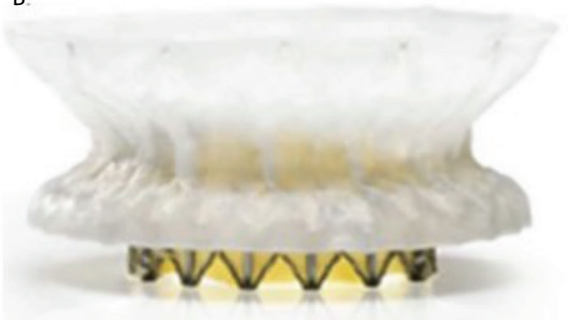

E

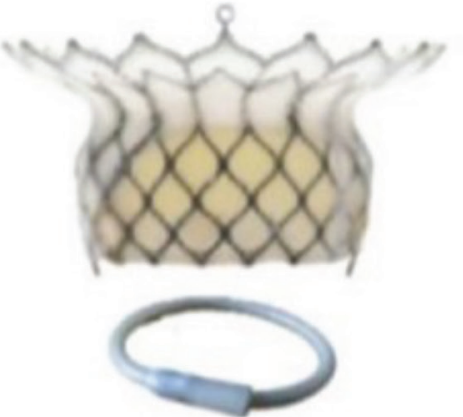

C
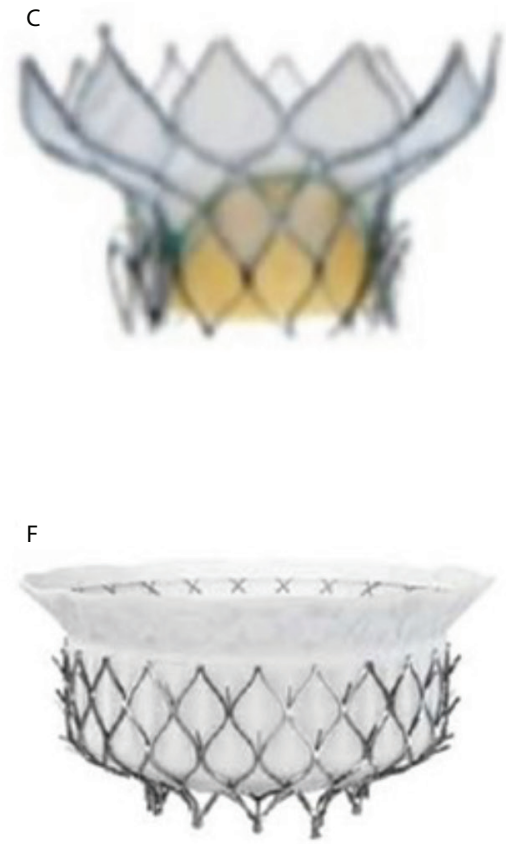

G

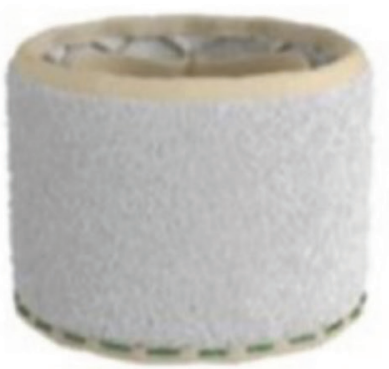

$\mathrm{H}$

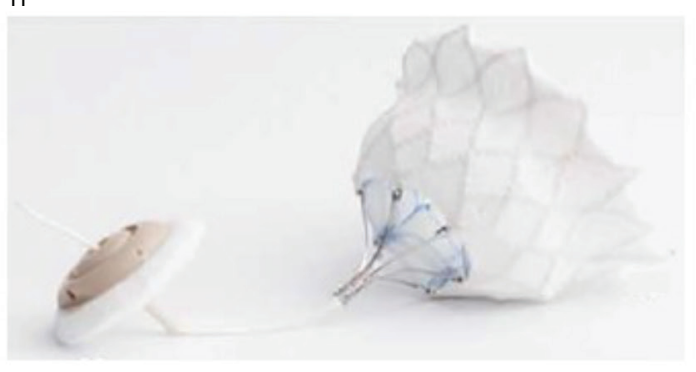

A: AltaValve тм (4C Medical); B: Cephea (Abbott Vascular); C: Cardiovalve (Cardiovalve); D: EVOQUE (Edwards Lifesciences); E: HighLife ${ }^{\mathrm{TM}}$ (HighLife Medical); F: Intrepid TM (Medtronic); G: SAPIEN M3 (Edwards Lifesciences); H: Tendyne TM (Abbott Vascular). Reproduced with permission of the Journal of the American Heart Association, Inc., by Wiley. ${ }^{18}$

\section{Technical challenges of transcatheter mitral valve replacement}

In contrast to the aortic valve, the anatomy of the mitral valve is much more complex. The mitral valve consists of two asymmetric leaflets, a saddle-shaped annulus and a complex subvalvular apparatus including a network of chordae tendineae and papillary muscles. ${ }^{18}$ The mitral valve is located posteriorly to the aortic valve and separated anteriorly by the aorto-mitral continuity. Unlike the aortic annulus, the mitral annulus is dynamic and changes shape and size during the cardiac cycle. Furthermore, the mitral valve is affected by numerous heterogeneous disease processes, which affect the valve or surrounding structures differently and pose unique anatomic challenges and considerations when developing a TMVR system. ${ }^{19}$ Both the complex anatomy and heterogeneous valvular pathophysiology present significant challenges for valve delivery, positioning, anchoring and sealing.

The first technical challenge of a TMVR system is the location of the valve, which makes device delivery and positioning difficult."1 To deliver the valve transfemorally, transseptal access is needed. In order to orient the valve within the native mitral valve from the left atrium, the delivery system must be equipped with the ability to provide a high degree of flexion. This challenge is compounded by the need to deliver a much larger valve than an aortic valve, which makes the system bulky. ${ }^{11} \mathrm{~A}$ transapical approach overcomes this issue by providing a more direct route, but it is also more invasive. Experience with TAVRs has shown that a transapical approach is associated with substantially more morbidity and mortality than a transfemoral approach..$^{20}$ Although patients treated by a transapical approach were higher-risk patients, the differences in clinical outcomes persisted in propensity-matched comparisons that account for differences in cardiovascular risk factors. ${ }^{20}$

The second challenge is the anchoring and sealing of the prosthetic valve. Unlike the aortic valve, which is roughly circular and static, the mitral valve is saddle shaped, with a dynamic annulus. ${ }^{11}$ Furthermore, a TAVR valve anchors itself in a rigid calcified annulus, but the mitral valve is generally less calcified, and poor anchoring of the delivered valve increases the risk for device migration or embolization. ${ }^{11}$ Additionally, the presence of significant or asymmetric mitral annular calcification (MAC) may result in poor sealing, leading to paravalvular leak, as well as device migration or embolization. ${ }^{21}$ 
Table 1: Transcatheter mitral valve replacement device characteristics

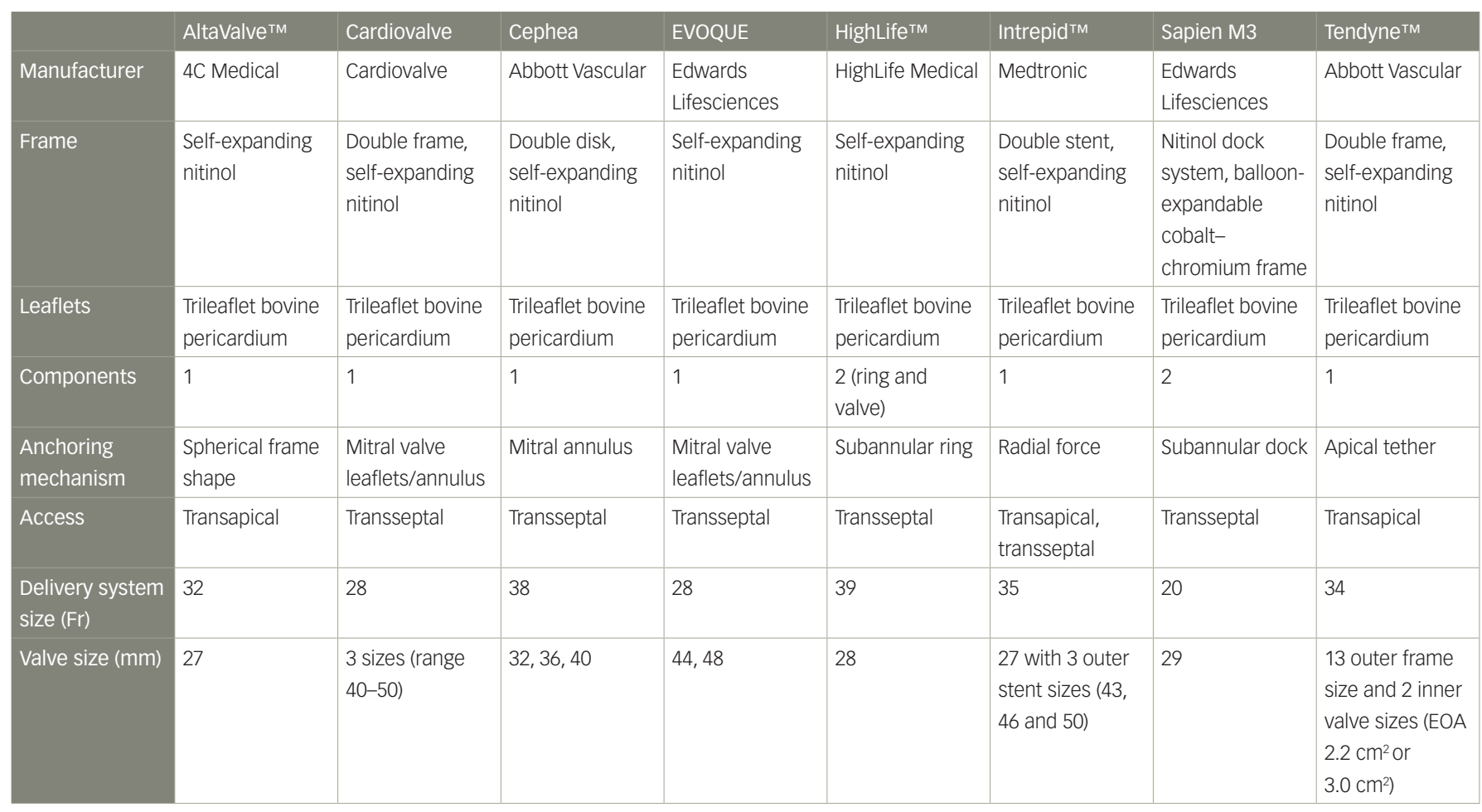

$E O A=$ effective orifice area.

Finally, the development of left ventricular outflow tract (LVOT) obstruction (LVOTO) following TMVR is a feared and potentially catastrophic complication. In a study by Yoon et al., $13.4 \%$ of patients who underwent TMVR developed LVOTO, and these patients had significantly higher procedural mortality compared with those without LVOTO (34.6\% versus 2.4\%; $p<0.001$ ). ${ }^{22}$ Several factors predict the risk for LVOTO, including aortomitral angle, septal hypertrophy, anterior mitral valve leaflet size and the shape of the left ventricle. ${ }^{21,22}$ Procedural risk factors include prosthesis positioning, inability to deploy the valve coaxially and device canting towards the LVOT. ${ }^{23}$ Computed tomography can be used to predict the risk of post-implant LVOTO by calculating the neo-LVOT, which is formed by the interventricular septum anteriorly and the displaced anterior mitral valve leaflet posteriorly. In the study by Yoon et al., a neo-LVOT $\leq 1.7 \mathrm{~cm}^{2}$ at end systole was considered high risk for the development of LVOTO.22

Over the past decade, various transapical and transseptal TMVR systems have been developed and investigated in early feasibility studies (Figure 1). ${ }^{18}$ Differences in TMVR systems are shown in Table 1. Although a transseptal delivery system is more appealing, clinical experience with this approach is eminently restricted compared with transapical systems. In the USA, none of the TMVR devices has been approved for commercial use.

\section{Transapical transcatheter mitral valve replacement systems under investigation}

\section{AltaValve ${ }^{\mathrm{TM}}$}

The AltaValve ${ }^{\mathrm{TM}}$ (4C Medical, Apple Grove, MN, USA) is unique in that it is a supra-annular system consisting of a 27-mm trileaflet bovine pericardial valve mounted into a self-expanding spherical nitinol frame. This frame self-anchors within the left atrium, with the valve sitting in a supra-annular position. ${ }^{24} \mathrm{~A}$ fabric skirt on the bottom of the frame at the level of the annular ring prevents paravalvular leaks. The unique position of the valve and frame minimizes the risk for LVOTO and embolization.
This design overcomes the anatomical challenges of other TMVR systems, which frequently cannot be implanted owing to unsuitable anatomy. Successful first-in-human experience has been reported. ${ }^{18}$ An early feasibility study (AltaValve early feasibility study protocol; ClinicalTrials.gov identifier: NCT03997305) is currently under way. ${ }^{25}$

\section{Tendyne ${ }^{\mathrm{TM}}$}

The Tendyne ${ }^{\mathrm{TM}}$ transcatheter mitral valve implantation system (Abbott Vascular, Santa Clara, CA, USA) includes a trileaflet pericardial valve in a dual-frame design with an anchoring system composed of an apical tether and pad. ${ }^{19}$ The outer frame is designed to conform to a wide range of mitral annular dimensions and provides a secure seal, while the inner frame houses the self-expanding valve. A left ventricular apical tethering system anchors the device. The device is fully repositionable and retrievable intraprocedurally. ${ }^{26}$

The Tendyne system was approved for commercial use in Europe in January 2020. The approval was based on a prospective, non-randomized, early feasibility study consisting of 100 patients with at least moderate-to-severe primary or secondary MR who were at high or prohibitive surgical risk. ${ }^{27}$ The mean age was $75.4 \pm 8.1$ years. The device was successfully implanted in $96 \%$ of patients. The 1-year survival was $72.4 \%$, with $84.6 \%$ of deaths due to cardiac causes. At 1 -year follow-up, there was significant clinical improvement, with $88.5 \%$ of patients reporting New York Heart Association (NYHA) class I or ॥ symptoms and $73.4 \%$ of patients reporting $\geq 10$-point improvement in Kansas City Cardiomyopathy Questionnaire (KCCQ) scores. After encountering six cases of valve thrombosis, the investigators implemented a protocol of warfarin with an international normalized ratio goal of 2.5-3.5 for at least 3 months. ${ }^{26}$

The ongoing SUMMIT trial (Clinical trial to evaluate the safety and effectiveness of using the Tendyne mitral valve system for the treatment 
of symptomatic mitral regurgitation; ClinicalTrials.gov identifier: NCT03433274) is a randomized controlled trial comparing the Tendyne TMVR system to TEER with MitraClip (Abbott Vascular, Chicago, IL, USA) in patients with symptomatic severe MR. ${ }^{27}$ In addition, the safety and efficacy of the Tendyne system in patients with severe MAC who are at prohibitive surgical risk will also be evaluated. The primary outcome is survival free of heart failure hospitalization at 12 months.

\section{Transseptal transcatheter mitral valve replacement systems under investigation Cardiovalve}

The Cardiovalve (Cardiovalve, or Yehuda, Israel) is a self-expanding trileaflet bovine pericardial valve mounted in a dual nitinol frame. ${ }^{21}$ The valve structure is modelled on an established surgical mitral valve, which has been adapted for TMVR. It is available in three sizes (range 40-50 mm). The delivery system is low profile and utilizes a multi-steerable catheter for coaxial implantation. The valve is anchored into the mitral annulus with 24 grasping legs, which minimizes atrial protrusion, and its low profile reduces the risk of left ventricular interference. ${ }^{21}$ The AHEAD European (AHEAD: European feasibility study of the Cardiovalve transfemoral mitral valve system; ClinicalTrials. gov identifier: NCT03339115) and AHEAD US (Cardiovalve transfemoral mitral valve system; ClinicalTrials.gov identifier: NCT03813524) trials will evaluate the safety and device performance of the Cardiovalve system. ${ }^{28,29}$ Data on the first five patients in the AHEAD European study were presented at the Transcatheter Cardiovascular Therapeutics (TCT) conference in 2018 (San Diego, CA, USA, 21-25 September 2018). There was $100 \%$ technical success, with no MR in $80 \%$ of patients and no LVOTO in any of the patients. ${ }^{30}$

\section{Cephea}

The Cephea TMVR system (Abbott Vascular, Chicago, IL, USA) consists of a self-expanding nitinol double-disk design with an outer ring that conforms to variable anatomy and an inner ring that houses a trileaflet bovine pericardial valve. ${ }^{31}$ It is available in three sizes $(32,36$ and $40 \mathrm{~mm})$. The device is both repositionable and recapturable. The valve anchors by axial compression forces in contrast to subvalvular anchoring systems. The low profile of the frame structure minimizes the risk for LVOTO. An early feasibility study included three patients at prohibitive surgical risk who underwent TMVR with the Cephea system. The valve was implanted successfully in all patients. ${ }^{32}$ Following implantation, all patients had, at most, mild MR and there was no clinically significant LVOTO. ${ }^{32}$ These haemodynamic parameters were maintained at 6 months, with mild paravalvular regurgitation in all patients. All patients reported NYHA class II symptoms and an improvement in KCCQ scores. ${ }^{31}$ To our knowledge, a total of seven patients have been treated with the device to date, and the results are awaited.

\section{EVOQUE}

The EVOQUE valve (Edwards Lifesciences, Irvine, CA, USA) is a trileaflet bovine pericardial valve on a nitinol frame with an intra-annular sealing skirt to decrease the risk of paravalvular leak. ${ }^{33}$ It is available in two sizes (44 mm and $48 \mathrm{~mm}$ ). The delivery system is low profile to minimize ventricular projection and LVOTO, and allows for multiple planes of flexion and independent depth control. The first-in-human experience using the EVOQUE TMVR system included 14 patients with severe MR who were at high or prohibitive surgical risk. ${ }^{33}$ Technical success was achieved in $13 / 14$ patients (92.9\%), with one conversion to open surgery due to severe paravalvular leak. One patient developed LVOTO. At 30 days, 13/14 patients were alive and one patient had died from pneumonia. MR was mild or less in all patients at 30 days, which included two patients who underwent paravalvular leak closure. The median KCCQ score improved by 22 points, and the majority of patients (81.8\%) had NYHA class II symptoms or less compared with $35.7 \%$ at baseline. ${ }^{33}$ The ongoing MISCEND trial (Edwards EVOQUE EOS MISCEND study; ClinicalTrials.gov identifier: NCT02718001) will further investigate the safety and efficacy of the EVOQUE system. ${ }^{34}$

\section{HighLife ${ }^{\text {TM }}$}

HighLife ${ }^{\mathrm{TM}}$ (HighLife Medical, Paris, France) is a two-component system that uses a 'valve-in-ring' concept, which theoretically reduces the risk for paravalvular leak and LVOTO. ${ }^{21}$ First, a subannular ring is implanted through a transarterial retrograde route. This ring acts as an anchor for the self-expanding trileaflet bovine pericardial valve. The valve is available in a single size $(28 \mathrm{~mm})$, but can accommodate an annular range of $32-48 \mathrm{~mm}$. The valve is deployed transseptally and selfcentres within the subannular ring. The first-in-human experience was presented at the 2017 TCT conference and described 15 patients who underwent TMVR with HighLife. ${ }^{31}$ Successful implantation was achieved in $13 / 15$ patients (87\%) and two patients converted to surgery. MR was trace in all successful implantations. An early feasibility study is currently under way (HighLife ${ }^{\mathrm{TM}}$ transcatheter mitral valve replacement system study; ClinicalTrials.gov identifier: NCT02974881). ${ }^{36}$

\section{Intrepid ${ }^{\mathrm{TM}}$}

IntrepidTM TMVR (Medtronic Inc., Minneapolis, MN, USA) is a 27-mm trileaflet bovine pericardial valve in a self-expanding nitinol frame with a dual-structure design, consisting of an outer fixation ring to engage the mitral annulus and a circular inner stent frame to house the valve. ${ }^{37}$ The outer fixation ring comes in three sizes $(43,46$ and $50 \mathrm{~mm}$ ) and is designed to accommodate the dynamic variability of the mitral annulus. ${ }^{19}$ It is delivered transapically or transseptally via a 35-Fr sheath.

The Intrepid Global Pilot study enrolled 50 patients with severe MR at high or extreme surgical risk. ${ }^{38}$ The device was successfully implanted by transapical approach in 48/50 patients (96\%). The 30-day mortality was $14 \%$ and no patient required repeat intervention. At a median follow-up of about 6 months, there was mild or no residual MR in all patients and significant improvement in symptoms: 79\% reported NYHA functional class I or || symptoms and there was significant improvement in the Minnesota Living with Heart Failure Questionnaire scores. ${ }^{38}$

The early feasibility study for the Intrepid TMVR system using a transseptal approach was recently published. ${ }^{39}$ This study enrolled 15 patients with moderate-to-severe symptomatic MR at high surgical risk. The device was successfully implanted in 14/15 patients (93\%) and one patient converted to surgery. At 30 days, there were no deaths, strokes or reinterventions. All implanted patients had trace or no residual MR and a mean gradient of $4.7 \pm 1.8 \mathrm{mmHg} .{ }^{39}$

The APOLLO trial (Transcatheter mitral valve replacement with the Medtronic IntrepidTM TMVR system in patients with severe symptomatic mitral regurgitation; ClinicalTrials.gov identifier: NCT03242642) is an ongoing, large, prospective, non-randomized trial evaluating patients with severe MR treated with Intrepid TMVR. ${ }^{40}$ The study consists of two cohorts: the primary cohort includes patients with primary or secondary MR without MAC and the second cohort includes those with MAC. The primary outcome is all-cause mortality or heart failure hospitalization at 30 days or KCCQ improvement $<10$ points. 


\section{SAPIEN M3}

The SAPIEN M3 (Edwards Lifesciences, Irvine, CA, USA) consists of a nitinol docking system with a 29-mm balloon-expandable bovine pericardial valve mounted on a cobalt-chromium stent frame. The valve is a modified SAPIEN 3 valve with an additional knitted polyethylene terephthalate skirt to aid sealing. Through a transseptal deflectable sheath placed in the left atrium, the docking system is advanced to the left ventricle and encircles the chordae tendineae. This dock facilitates anchoring of the valve, which is deployed inside the dock. ${ }^{41}$ The results of the first 10 patients who received this valve have been published. ${ }^{41}$ The device was successfully implanted in 9/10 patients, with a reduction of MR severity to trivial in all patients and a mean transmitral gradient of $2.3 \mathrm{mmHg}$. At 30 days, there was no stroke, myocardial infarction, device migration, embolization, LVOTO, rehospitalization or conversion to mitral surgery. ${ }^{40}$ One patient had recurrent regurgitation due to a paravalvular leak, which was treated with a closure device. In a subsequent early feasibility trial, 35 patients underwent TMVR, with an $88.6 \%$ technical success rate. ${ }^{42}$ The 30-day survival rate was $97.1 \%$ and $87.9 \%$ had $\leq 1+$ MR. ${ }^{42}$

\section{Conclusion}

TMVR is a promising alternative to surgical intervention for the treatment of severe MR, particularly in patients at high or prohibitive surgical risk. However, designing a widely applicable TMVR system is challenging owing to numerous anatomic, patient-specific and engineering-related considerations. As a result of these complexities and the heterogeneity of mitral valve disease, it is unclear whether there will be a universal TMVR system suitable for all patients, or if there will be multiple unique platforms from which the operator will select the best suited for each individual patient. In addition to procedural technicalities, deliberate planning with multimodality imaging and careful patient selection based on anatomic and clinical features will be critical in achieving success. ${ }^{1,11,22}$ Although many TMVR systems utilize a transapical approach, the transition of these systems to a transseptal delivery may be critical to their success. Initial experience with numerous TMVR systems has shown early promise, and through incremental improvements of newer-generation devices, TMVR will likely emerge as a real option to treat MR.
1. Otto CM, Nishimura RA, Bonow RO, et al. 2020 ACC/AHA Guideline for the management of patients with valvular hear disease: a report of the American College of Cardiology/ American Heart Association Joint Committee on Clinical Practice Guidelines. Circulation. 2021;143:e72-227.

2. Popma JJ, Adams DH, Reardon MJ, et al. Transcatheter aortic valve replacement using a self-expanding bioprosthesis in patients with severe aortic stenosis at extreme risk for surgery. J Am Coll Cardiol. 2014:63:1972-81.

3. Popma JJ, Deeb GM, Yakubov SJ, et al. Transcatheter aortic-valve replacement with a self-expanding valve in low-risk patients. N Engl J Med. 2019:380:1706-15.

4. Reardon MJ, Van Mieghem NM, Popma JJ, et al. Surgical or transcatheter aortic-valve replacement in intermediate-risk patients. N Eng/ / Med. 2017:376:1321-31.

5. Adams DH, Popma JJ, Reardon MJ, et al. Transcatheter aortic-valve replacement with a self-expanding prosthesis N Engl J Med. 2014;370:1790-8.

6. Leon MB, Smith CR, Mack M, et al. Transcatheter aortic-valve implantation for aortic stenosis in patients who cannot undergo surgery. N Engl I Med. 2010;363:1597-607.

7. Leon MB, Smith $C R$, Mack MJ, et al. Transcatheter or surgical aortic-valve replacement in intermediate-risk patients. N Eng/」 Med. 2016;374:1609-20.

8. Mack MJ, Leon MB, Thourani VH, et al. Transcatheter aortic-valve replacement with a balloon-expandable valve in low-risk pationts. N Eng/J Med 2019:380:1695-705.

2. Nkomo VT, Gardin JM, Skelton TN, et al. Burden of valvular heart Nkomo VI, Gardin JM, Skelton TN, et al. Burden of valvular heart

diseases: a population-based study. Lancet. 2006,368.1005-11. . Coffey S, Cairns BJ, lung B. The modern epidemiology
valve disease. Heart Br Card Soc. 2016;102:75-85.

11. Fiorilli PN, Herrmann HC, Szeto WY. Transcatheter mitral valve replacement: latest advances and future directions. Ann Cardiothorac Surg. 2021;10:855-95.

12. Goel SS, Bajaj N, Aggarwal B, et al. Prevalence and outcomes of unoperated patients with severe symptomatic mitral regurgitation and heart failure: comprehensive analysis to determine the potential role of MitraClip for this unmet need. J Am Coll Cardiol. 2014;63:185-6.

13. Feldman T, Foster E, Glower DD, et al. Percutaneous repair or surgery for mitral regurgitation. N Eng/ J Med. 2011:364:1395-406

14. Stone GW, Lindenfeld J, Abraham WT, et al. Transcatheter mitral-valve repair in patients with heart failure. $N$ Eng/ I Med. 2018:379:2307-18

15. Witte KK, Lipiecki J, Siminiak T, et al. The REDUCE FMR Trial: a randomized sham-controlled study of percutaneous mitral annuloplasty in functional mitral regurgitation. JACC Heart Fail. 2019;7:945-55.

16. Giallauria F, Di Lorenzo A, Parlato A, et al. Individual patient data meta-analysis of the effects of the CARILLON ${ }^{\circledast}$ mitral contour system. ESC Heart Fail. 2020;7:3383-91.
17. Messika-Zeitoun D, Nickenig G, Latib A, et al. Transcatheter mitral valve repair for functional mitral regurgitation using the Cardioband system: 1 year outcomes. Eur Heart 2019;40:466-72

18. Testa L, Popolo Rubbio A, Casenghi M, et al. Transcatheter mitral valve replacement in the transcatheter aortic valve replacement era. J Am Heart Assoc. 2019;8:e013352.

19. Regueiro A, Granada JF, Dagenais $F$, Rodés-Cabau J. Transcatheter mitral valve replacement: insights from early clinical experience and future challenges. I Am Coll Cardiol. 2017:69:2175-92.

20. Blackstone EH, Suri RM, Rajeswaran J, et al. Propensity-matched comparisons of clinical outcomes after transapical or transfemoral transcatheter aortic valve replacement Circulation 2015:131:1989-2000.

21. Hensey M, Brown RA, Lal $S$, et al. Transcatheter mitral valve replacement: an update on current techniques, technologies, and future directions. JACC Cardiovasc Interv. 2021;14:489-50

22. Yoon S-H, Bleiziffer S, Latib A, et al. Predictors of left ventricular outflow tract obstruction after transcatheter mitral valve replacement. JACC Cardiovasc Interv. 2019;12:182-93.

23. Weir-McCall JR, Blanke P, Naoum C, et al. Mitral valve imaging with CT: relationship with transcatheter mitral valve interventions. Radiology. 2018:288:638-55.

24. Goel SS, Zuck V, Christy J, et al. Transcatheter mitral valve therapy with novel supra-annular AltaValve: first experience in the United States. JACC Case Rep. 2019;1:761-4.

25. ClinicalTrials.gov. AltaValve Early Feasibility Study Protocol. ClinicalTrials.gov Identifier: NCT03997305. Available at: https://clinicaltrials.gov/ct2/show/NCT03997305 (accessed 3 December 2021).

26. Sorajja P, Moat N, Badhwar V, et al. Initial feasibility study of a new transcatheter mitral prosthesis: the first 100 patients. J Am Coll Cardiol. 2019;73:1250-60

27. ClinicalTrials.gov. Clinical Trial to Evaluate the Safety and Effectiveness of Using the Tendyne Mitral Valve System for the Treatment of Symptomatic Mitral Regurgitation (SUMMIT). ClinicalTrials.gov Identifier: NCT03433274. Available at: https://clinicaltrials.gov/ct2/show/NCT03433274 (accessed 3 December 2021).

28. ClinicalTrials.gov. AHEAD: European Feasibility Study of the Cardiovalve Transfemoral Mitral Valve System (AHEAD. ClinicalTrials.gov Identifier: NCT03339115. Available at: https://clinicaltrials.gov/ct2/show/NCTO3339115 (accessed 3 December 2021).

29. ClinicalTrials.gov. Cardiovalve Transfemoral Mitral Valve System (AHEAD). ClinicalTrials.gov Identifier: NCT03813524. Available at: https://clinicaltrials.gov/ct2/show/NCT03813524 (accessed 3 December 2021)

30. Maisano F. Cardiovalve: device attributes, implant procedure, and early results. Presented at: Transcatheter Cardiovascular Therapeutics, San Diego, CA, USA, 24 September 2018.
Available at: www.tctmd.com/slide/cardiovalve-deviceattributes-implant-procedure-and-early-results (accessed 15 December 2021

31. Modine $T$, Vahl $T P$, Khalique OK, et al. First-in-human implant of the Cephea transseptal mitral valve replacement system. Circ Cardiovasc Interv. 2019;12:e008003.

32. Alperi A, Dagenais F, Del Val D, et al. Early experience with a novel transfemoral mitral valve implantation system in complex degenerative mitral regurgitation. JACC Cardiovasc Interv. 2020:13:2427-37.

33. Webb J, Hensey M, Fam N, et al. Transcatheter mitral valve replacement with the transseptal EVOQUE System. JACC Cardiovasc Interv. 2020:13:2418-26.

34. ClinicalTrials gov. Edwards EVOQUE EOS MISCEND Study. ClinicalTrials.gov Identifier: NCT02718001. Available at: https://clinicaltrials.gov/ct2/show/NCT02718001 (accessed 3 December 2021).

35. Piazza N. Established TMVR5: HighLife - device description, strengths and weaknesses, and updated summary outcomes. Presented at: Transcatheter Cardiovascular Therapeutics. Denver, CO, USA, 31 October 2017. Avallable at: www.tctmd. com/slide/established-tmvr-5-highlife-device-descriptionstrengths-and-weaknesses-and-updated-summary (accessed 15 December 2021)

36. ClinicalTrials.gov. HighLife ${ }^{\mathrm{TM}}$ Transcatheter Mitral Valve Replacement System Study. ClinicalTrials.gov Identifier: NCT02974881. Available at: https://clinicaltrials.gov/ct2/show/ NCT02974881 (accessed 3 December 2021).

37. McCarthy PM, Kislitsina ON, Malaisrie SC, Davidson C Transcatheter mitral valve replacement with Intrepid. Inter Cardiol Clin 2019;8:287-94.

38. Bapat V, Rajagopal V, Meduri C, et al. Early experience with new transcatheter mitral valve replacement. J Am Coll Cardid 2018;71:12-21

39. Zahr F, Song HK, Chadderdon SM, et al. Thirty-day outcomes following transfemoral transseptal transcatheter mitral valve replacement: Intrepid TMVR early feasibility study results. JACC Cardiovasc Interv. 2021;S1936-8798(21)01910-5.

40. ClinicalTrials.gov. Transcatheter Mitral Valve Replacement with the Medtronic IntrepidTM TMVR System in Patients with Severe Symptomatic Mitral Regurgitation (APOLO) ClinicalTrials gov symtifier: NCT03242642. Ava able at: https:/clinicatrias.gov/ ct2/s

ct2/shor

. Web JG, Murdoch DJ, Boone RH, et al. Percutaneous transcatheter mitral valve replacement: first-in-human 2019:73:1239-46.

42. Makkar R, O'Neill William, Whisenant B, et al. TCT-8 updated 30-day outcomes for the U.S. early feasibility study of the SAPIEN M3 transcatheter mitral valve replacement system. J Am Coll Cardiol. 2019;74(Suppl. 13):B8. 